\title{
SAÚDE COLETIVA NA FORMAÇÃO ACADÊMICA: EXPERIÊNCIAS VIVIDAS NOS COMPONENTES CURRICULARES DO CURSO DE ODONTOLOGIA DA UNIVERSIDADE ESTADUAL DE FEIRA DE SANTANA (2013-2018)
}

\author{
COLLECTIVE HEALTH IN THE ACADEMIC FORMATION: EXPERIENCES LIVED \\ IN CURRICULAR COMPONENTS OF THE DENTISTRY COURSE OF THE STATE \\ UNIVERSITY OF FEIRA DE SANTANA (2013-2018)
}
Ana Áurea Alécio de Oliveira Rodrigues, Brisa Oliveira Silva Vitória, Cassia Tainar da Silva Souza, Leonardo de Jesus Costa Santos, Letícia Santos Souza, Luana Gracia dos Santos, Taiane de Santana Azêvedo Falcão, Vinicius Silva Bastos de Jesus

Universidade Estadual de Feira de Santana - UEFS

\begin{abstract}
This article aims to report the experience of students who entered the Odontology course of the Feira de Santana State University - UEFS in the academic semester of 2013.2, in the curricular components of Public Health throughout the graduation. Collective Health is part of the compulsory academic activities since the beginning of the course. In 2011 the curricular matrix underwent a process of reformulation and the academic formation has undergone changes that emphasize the necessity of the contents that allow to act in the public health services. The experience in family health units since the second semester has allowed students to understand that the care and planning of each patient is not only individual, reflected in their complaint, in the perspective of a result, but rather that the historical context social environment in which he is involved says much about his state of heal.
\end{abstract}

Key words: Plublic Health; Curriculum; Dentistry.

\section{Resumo}

Este artigo tem como objetivo relatar a experiência dos estudantes que ingressaram no curso de Odontologia da Universidade Estadual de Feira de Santana - UEFS no semestre letivo de 2013.2, nos componentes curriculares da Saúde Coletiva durante toda a graduação. A Saúde Coletiva está inserida nas atividades acadêmicas obrigatórias desde o início do curso. Em 2011 a matriz curricular passou por um processo de reformulação e a formação acadêmica vem sofrendo mudanças que enfatizam a necessidade dos conteúdos que permitam atuar nos serviços de saúde públicos. A vivência nas unidades de saúde da família, desde o segundo semestre, possibiliou aos estudantes compreender que o atendimento e planejamento de cada paciente não é apenas de caráter individual, refletido na sua queixa, na perspectiva diante de um resultado, mas que o contexto histórico social em que está envolvido diz muito sobre seu estado de saúde.

Palavras-chave: Saúde Coletiva; Currículo; Odontologia. 


\section{Introdução}

A Atenção Básica constitui um conjunto de ações de saúde, no âmbito individual ou coletivo, que abrange a promoção e proteção da saúde, a prevenção de agravos, o diagnóstico, o tratamento, a reabilitação e a manutenção da saúde, situadas no primeiro nível de atenção do sistema de saúde. É desenvolvida por meio do exercício de práticas gerenciais e sanitárias democráticas e participativas, sob forma de trabalho em equipe, dirigidas à populações de territórios bem delimitados, pelas quais assume a responsabilidade sanitária, considerando a dinamicidade existente no território em que vivem essas populações'.

Neste contexto, utiliza tecnologias de elevada complexidade e baixa densidade, que devem resolver os problemas de saúde de maior frequência e relevância em seu território. Orienta-se pelos princípios da universalidade, da acessibilidade e da coordenação do cuidado, do vínculo e continuidade, da integralidade, da responsabilização, da humanização, da equidade e da participação social ${ }^{2}$.

$\mathrm{Na}$ organização da Atenção Básica, um aspecto fundamental é o conhecimento do território, que não pode ser compreendido apenas como um espaço geográfico, delimitado para constituir a área de atuação dos serviços. Ao contrário, deve ser reconhecido como "Espaço Social" onde, ao longo da história, a sociedade foi se constituindo e, por meio do processo social de produção, dividindo-se em classes diferenciadas, com acessos também diferenciados aos bens de consumo, incluídos os serviços de saúde. Assim, conhecer o território implica em um processo de reconhecimento e apropriação do espaço local e das relações da população da área de abrangência com a unidade de saúde, levando em consideração dados como perfil demográfico e epidemiológico da população, contexto histórico e cultural, equipamentos sociais (associações, igrejas, escolas, creches...), lideranças locais, e outros considerados relevantes para intervenção no processo saúde-doença'.

Os primeiros modelos de atenção em saúde bucal no Brasil demonstraram ineficácia, na medida em que não responderam, em níveis significativos, aos problemas de saúde da população, e ineficiência, uma vez que possuíam um alto custo de execução e baixíssimo rendimento, apesar do grande desenvolvimento científico e tecnológico da Odontologia nos últimos anos. Historicamente, esses modelos deixaram traços marcantes na prática odontológica atual. Antes da Constituição Federal de 1988 e da criação do Sistema Único de Saúde (SUS), só tinham direito a assistência odontológica pública os trabalhadores contribuintes ao INAMPS (Instituto Nacional de Assistência Médica da Assistência Social), a exemplo do que também acontecia em outros setores da saúde. Essa assistência priorizava as ações curativas, restritas e isoladas, em nível ambulatorial e de livre demanda, realizada individualmente pelo Cirurgião Dentista (CD). Com o SUS, o acesso universal da população aos serviços de saúde foi garantido legalmente e não mais se admite exclusão de grupos populacionais ${ }^{3}$.

A proposição pelo Ministério da Saúde das diretrizes para uma Política Nacional de Saúde Bucal e de sua efetivação, por meio do Brasil Sorridente, tem, na Atenção Básica, um de seus mais importantes pilares. Organizar as ações no nível da Atenção Básica é o primeiro desafio a que se lança o Brasil Sorridente, na certeza de que sua consecução significará a possibilidade de mudança do modelo assistencial no campo da saúde bucal'.

O Programa Brasil Sorridente foi criado em 2004 quando foi lançada a Política Nacional de Saúde Bucal. Seu principal objetivo é a reorganização da prática e a qualificação das ações e serviços oferecidos, reunindo uma série de ações em saúde bucal, com ampliação do acesso ao tratamento odontológico gratuito aos brasileiros por meio do Sistema Único de Saúde (SUS). As principais linhas de ação do programa são: reorganização da Atenção Básica em saúde bucal, principalmente com a implantação das Equipes de Saúde Bucal na Estratégia Saúde da Família e ampliação e qualificação da Atenção Especializada, especialmente com a 
implantação de Centros de Especialidades Odontológicas e Laboratórios Regionais de Próteses Dentárias ${ }^{4}$.

A Resolução CNE/CES 3, de 19 de Fevereiro de 2002, do Conselho Nacional de Educação institui Diretrizes Curriculares Nacionais do Curso de Graduação em Odontologia. Em seu Art. 70 trata que a formação do Cirurgião Dentista deve garantir o desenvolvimento de estágios curriculares, sob supervisão docente. Este estágio deverá ser desenvolvido de forma articulada e com complexidade crescente ao longo do processo de formação. A carga horária mínima do estágio curricular supervisionado deverá atingir $20 \%$ da carga horária total do Curso de Graduação em Odontologia proposto, com base no Parecer/Resolução específico da Câmara de Educação Superior do Conselho Nacional de Educação ${ }^{5}$.

Nessas Diretrizes fica evidente a preocupação com o estabelecimento de uma articulação entre a educação superior e a saúde, objetivando a formação de profissionais com ênfase na promoção, prevenção, recuperação e reabilitação da saúde, de acordo com os princípios e diretrizes do Sistema Único de Saúde (SUS). Com base nessas Diretrizes Curriculares, observa-se uma indução ao processo de reforma curricular nos cursos de graduação em Odontologia. Como também é perceptível que as políticas de saúde, a inserção da saúde bucal na ESF e as questões do mercado de trabalho instigam um novo olhar sobre a prática odontológica ${ }^{3}$.

A sala de aula não se limita a um espaço físico dentro da universidade. O aluno precisa ser inserido no contexto profissional, saindo de práticas realizadas exclusivamente dentro do espaço físico formal para atividades extramuros. As atividades extramuros têm sido utilizadas com sucesso entre os graduandos dos cursos da saúde, através do exercício de práticas educativas e preventivas na comunidade, em parcerias públicas e privadas. É uma variação do ambiente ensino/aprendizagem valiosa tanto pelo contato do aluno com a comunidade, quanto pela grande interação entre professor e aluno. Os cursos de saúde devem proporcionar a aquisição de conhecimentos, habilidades e atitudes na vivência de um mundo real, conhecida através da integração da universidade com a comunidade. $\mathrm{O}$ aluno em contato com comunidades carentes, além do aprendizado, exerce cidadania, tornando-se um profissional mais humano ${ }^{6}$.

Pesquisadores afirmam que algumas características quanto ao perfil desejável para o profissional de Odontologia que busca trabalhar no Programa de Saúde da Família PSF seria o de enxergar o paciente em sua totalidade biopsicossocial e não de forma fragmentada, sabendo integrar-se com os outros profissionais envolvidos na equipe do PSF, apresentando sensibilidade para as questões sociais, estabelecendo vínculos e criando laços de compromisso entre os profissionais de saúde e a comunidade, humanizando as práticas de saúde e tendo uma visão ampliada do processo saúde/doença. Mecanismos diversos têm sido propostos para possibilitar o desenvolvimento de sensibilidade social entre os acadêmicos. Entre eles, podem ser citados, o envolvimento do estudante de Odontologia junto a projetos de inserção comunitária ou trabalhos extramuros em conjunto com estudos dos processos saúdedoença junto à comunidade. Presume-se que o ensino de Odontologia, ao incorporar a realidade social, contribui para a formação de profissionais dentro da perspectiva que exige a ESF 7 .

Um estudo realizado por Almeida (2012) teve como objetivo avaliar sob o ponto de vista dos usuários a inserção do estudante de graduação na atenção básica. Neste trabalho, constatou-se a contribuição positiva da inserção do aluno de graduação nas Unidades Básicas de Saúde na opinião dos próprios usuários do serviço, visto que, com essa inserção comunitária, os estudantes tornam-se conhecedores e potenciais modificadores da realidade. Além disso, o julgamento favorável dos usuários em relação à presença e colaboração dos alunos em atividades voltadas à humanização, ao cuidado e à qualificação da atenção à saúde torna-se um subsídio para a formação de profissionais críticos, com consciência da realidade social na qual estão inseridos e 
com capacidade para desenvolver ações voltadas à prevenção, promoção e educação em saúde, ou seja, profissionais socialmente referenciados e comprometidos ${ }^{8}$.

Leme $(2015)^{9}$, em seu estudo buscou analisar as percepções de graduandos de um curso de odontologia em relação à importância atribuída ao estágio realizado em Unidades de Saúde da Família (USF) para sua formação profissional. Observou-se que os graduandos atribuíram importância positiva ao estágio nas USF, para a sua formação profissional. A análise qualitativa e quantitativa apontou que estes foram capazes de valorizar a vivência prática no serviço, o conhecimento da realidade social, o contato com profissionais de outras áreas e o treinamento técnico em condições distintas daquelas encontradas na faculdade, embora tenham demonstrado preocupação em relação à interferência na produção da clínica intramuros ${ }^{9}$.

Em Feira de Santana (BA), localizada na região Centro-Norte baiana e distante 115 quilômetros da capital (Salvador), segunda maior cidade do estado da Bahia em porte populacional, a implantação do PSF pela Prefeitura Municipal de Feira de Santana foi iniciada em novembro de 2000. Foram instaladas duas Unidades de Saúde Familiar (USF) - correspondendo, à época, a menos de $2 \%$ de cobertura da população feirense -, como Projeto Piloto, no bairro do Novo Horizonte. Desde 2004, encontra-se na Gestão Plena do Sistema Municipal de Saúde. Sua rede de serviços de Atenção Básica à Saúde ( $A B S$ ) conta com 72 equipes de Saúde da Família, mas apenas 30 possuem equipe de saúde bucal ${ }^{10}$. A Universidade Estadual de Feira de Santana - UEFS conta com essas 30 Unidades de Saúde da Família que possuem equipes de saúde bucal para a realização dos estágios de campo dos estudantes do curso de Odontologia da instituição.

Este artigo tem como objetivo relatar a experiência dos estudantes do 10 o semestre letivo de 2018.2 do Curso de Odontologia da UEFS na saúde coletiva durante toda a graduação.

\section{Metodologia}

Este trabalho apresenta a vivência acadêmica na Atenção Primária a Saúde do 1 ㅇ ao 10 ㅇ semestre, relatada por uma turma de estudantes do curo de Odontologia da UEFS. O ensino da instituição é diferenciado do ensino de universidades que não abordam ou retratam pouco o conteúdo de saúde pública no currículo. A Saúde coletiva está inserida nas atividades acadêmicas obrigatórias desde o início do curso, cuja matriz curricular passou por um processo de reformulação em 2011 e foi estruturada em quatro grandes áreas de conhecimento: Ciências Biológicas e da Saúde, Ciências Humanas e Sociais, Saúde Coletiva e Ciências Odontológicas. Os componentes curriculares foram organizados em Estágios em Saúde Coletiva e Saúde Bucal Coletiva, Estudos Integrados e Estágios em Clínica Odontológica Integrada.

Após a reorganização do currículo, a matriz curricular contempla 6 disciplinas que abordam a Saúde Coletiva, sendo elas: Saúde Coletiva em Odontologia (1ㅇ semestre), Estágio em Saúde Coletiva (2o semestre), Saúde Bucal Coletiva (3ㅇ semestre), Estágio em Saúde Bucal Coletiva I (4o semestre), Estágio em Saúde Bucal Coletiva II (5음 semestre) e Estágio em Saúde Bucal Coletiva III (10으 semestre). Além destas, a Saúde Coletiva também está inserida nas disciplinas de Estudos Integrados e nos Estágios em Clínica Odontológica Integrada. Os estudantes de Odontologia graduados na UEFS estão em contato com os preceitos da saúde coletiva e com a Atenção Primaria nas USF, do primeiro até o último semestre do curso.

\section{Relato de Experiência}

O nosso primeiro contato com uma Unidade Básica de Saúde (UBS) foi no Estágio em Saúde Coletiva I, onde a turma dividiu-se em dois grupos, um estava na UBS do bairro Feira VI e outro na USF do bairro Feira IX.

O grupo que estava na UBS do Feira VI desenvolvia atividades de visita domiciliar e terrirorialização no bairro Papagaio, coleta dos dados das fichas $A$ e $B$, interpretação, tabulação e elaboração de gráficos a partir das fichas. Com relação à estrutura física da UBS, esta era composta por 9 salas, com 
consultório odontológico e a equipe era formada por cirurgiã dentista, médico, enfermeira e 12 Agentes Comunitários de Saúde (ACS) que assistiam as áreas dos bairros Feira VI, Papagaio e Santa Quitéria. Aproximadamente 3.000 famílias eram cobertas pelo atendimento desta Unidade, totalizando cerca de 7.000 pessoas.

Durante as visitas a UBS do bairro Feira VI, houve várias tentativas de reuniões para planejar atividades nas áreas cobertas por esta, porém nenhuma obteve êxito, com isso foi necessário realocar o grupo que estava nesta UBS para a USF no distrito de Jaíba Feira de Santana. No mesmo período o grupo que estava realizando as atividades na USF do bairro Feira IX conseguiu fazer todas as reuniões de planejamentos das atividades propostas pela disciplina com a equipe de saúde, que englobava visitas domiciliares nas áreas cobertas, além da realização de coleta dos dados das fichas A e B, interpretação, tabulação e elaboração de gráficos a partir das fichas, porém a USF do bairro Feira IX entrou em reforma e ficou um período longo sem funcionamento, assim, esse grupo também foi transferido para outra USF no distrito de Terra Dura - Feira de Santana.

A equipe mostrou-se bem receptiva com as atividades desenvolvidas pelo estágio, contudo aconteceu a saída da cirurgiãdentista, o que fez o grupo migrar de USF mais uma vez, juntando-se ao grupo que pertencia a USF do Feira VI, que agora estava na USF de Jaíba.

A USF de Jaíba passou a ser o campo de estágio de toda a turma do 7으 ao 10응 semestre. As atividades realizadas pela equipe são de cunho curativo, educativo e preventivo. Os profissionais, juntamente com os discentes (Figuras 1 e 2) realizavam palestras, feiras de saúde, visita domiciliar e outras tarefas coletivas para abordar temas que interferem na qualidade de vida da população. É válido ressaltar a importância do trabalho dos agentes comunitários de saúde (ACS), uma vez que estes funcionam como elo entre os usuários e as unidades de saúde.

Figuras 1 e 2: Atividades realizadas na USF do distrito de Terra dura - Feira de Santana, Bahia, Brasil, 2016.
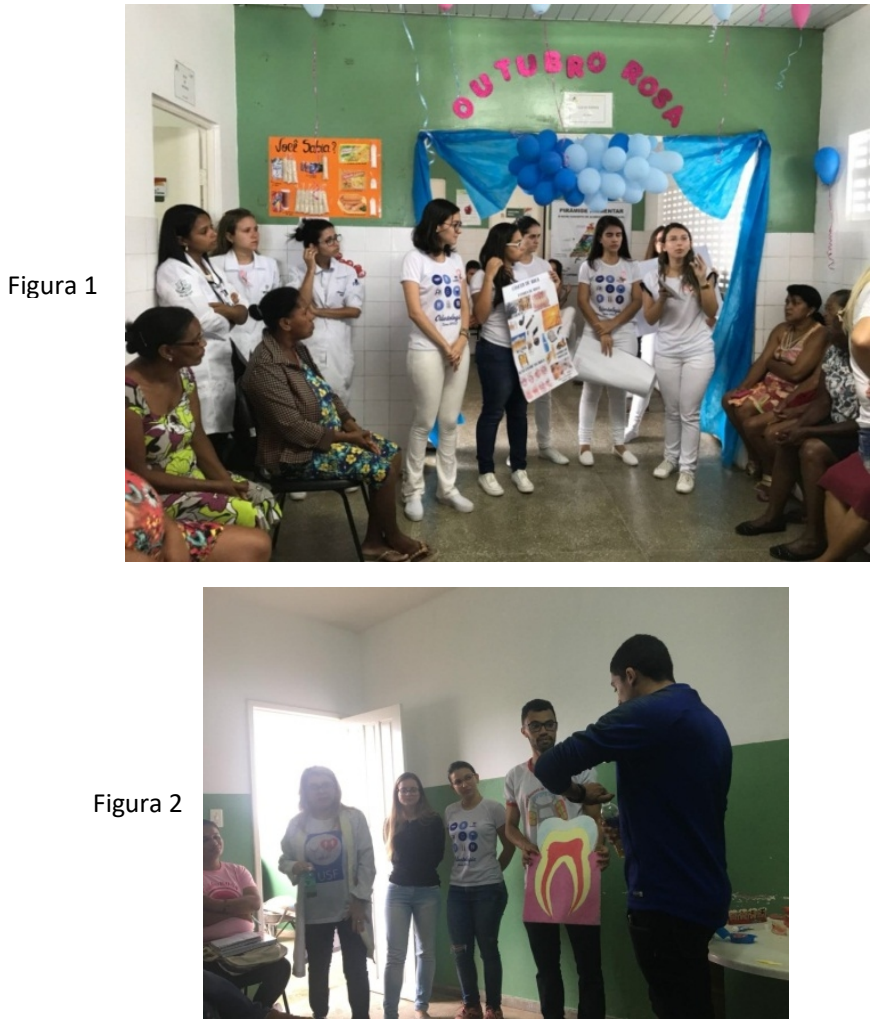

Fonte: acervo pessoal dos estagiários da turma 2013.2 
Nos Estágios realizados dentro das disciplinas Estágio em Clínica Odontológica Integrada - ECOI II, III e IV, as visitas à USF em Jaíba foram feitas por duplas, onde ocorriam palestras na sala de espera para os pacientes com os mais variados temas de saúde e o atendimento odontológico junto com 0 cirurgião-dentista da USF.
Além dessas atividades, em quase todos esses componentes curriculares eram desenvolvidas ações com a participação da comunidade adstrita à USF, como por exemplo, Feiras de Saúde, Oficinas de saúde bucal, café da manhã com grupo de idosos e também capacitação das ACS, sobre temas sugeridos pela própria equipe (Figura 3).

Figura 3: Capacitação das Agentes Comunitárias de Saúde da USF do distrito de Jaíba - Feira Santana, Bahia, Brasil, 2018.

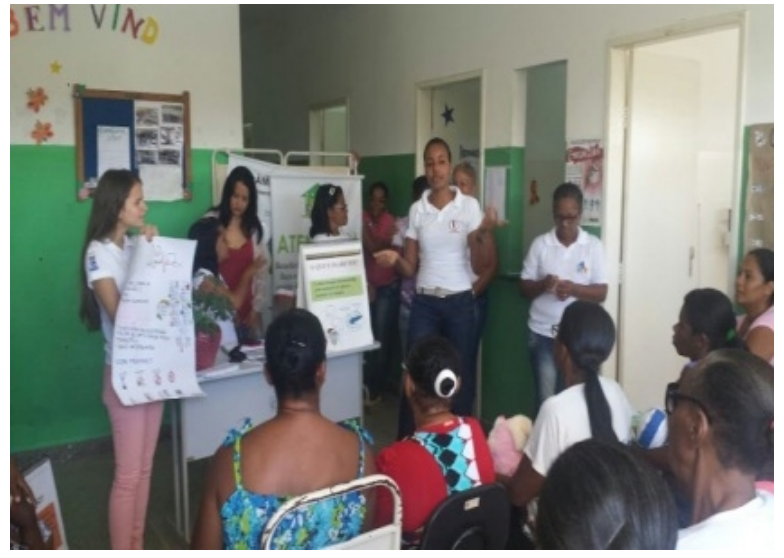

Fonte: acervo pessoal dos estagiários da turma 2013.2

Especificamente na ECOI IV foi realizada uma atividade em conjunto com outros alunos dos estágios de cursos e universidades diferentes, com o apoio do Núcleo Ampliado de Saúde da
Família (NASF). Dois momentos marcaram a ação ampliada, o Outubro Rosa e o Novembro Azul, com palestras, mesas demonstrativas, alongamentos e um café da manhã (Figuras 4 e 5 ).

Figuras 4 e 5: Atividades de sala de espera na campanha do outubro rosa e novembro azul realizada na USF do distrito de Jaíba - Feira de Santana, Bahia, Brasil, 2017.

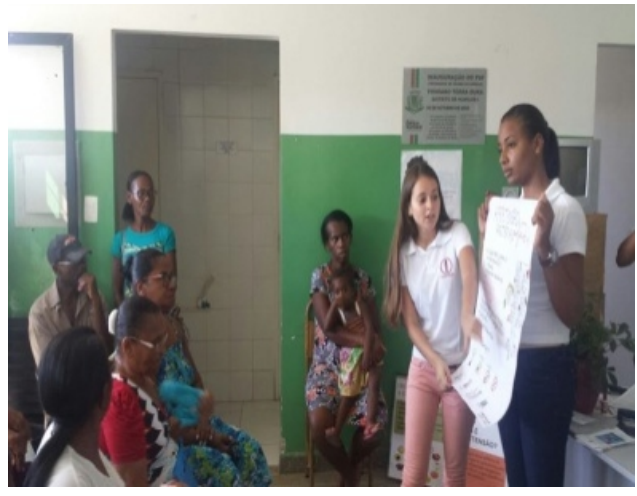

Figura 4

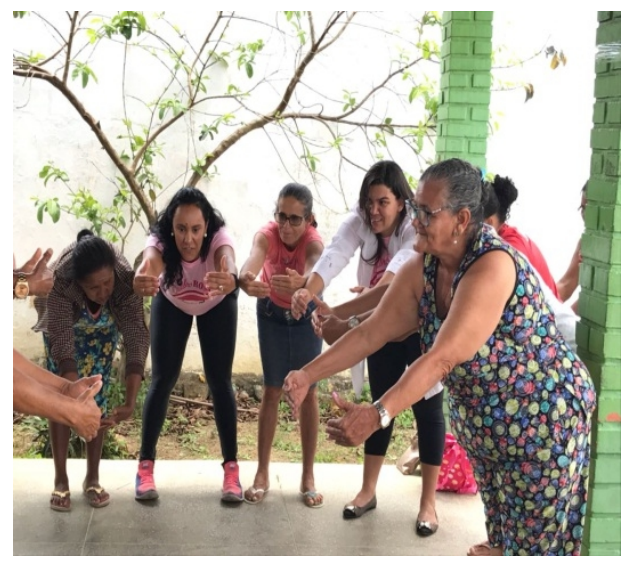

Figura 5

Fonte: acervo pessoal dos estagiários da turma 2013.2

SAÚDE COLETIVA NA FORMAÇÃO ACADÊMICA: EXPERIÊNCIAS VIVIDAS NOS COMPONENTES CURRICULARES DO CURSO DE ODONTOLOGIA DA UNIVERSIDADE ESTADUAL DE FEIRA DE SANTANA (2013-2018) Rev. Saúde.Com 2020; 16(4): 1999 - 2006. 
Na ECOI V ao invés de realizar visitas à USF, foram realizadas visitas ao Centro de Especialidades Odontológicas - CEO do bairro George Américo, onde os estudantes realizaram salas de espera com temas relacionados à saúde bucal e o atendimento clínico odontológico sob a supervisão do especialista do turno. As palestras de salas de espera sobre saúde bucal também eram realizadas antes do início dos atendimentos ambulatoriais na UEFS, nos turnos dos Estágios em Clínica Odontológica Integrada I, II, III, IV e V.

Nos componentes Estágio em Saúde Bucal I e II, Saúde Coletiva, Estágio em Saúde Bucal Coletiva I, II e III, foram realizadas reuniões com as USF em cada semestre para planejar as atividades que foram desenvolvidas ao longo de cada estágio, sempre se preocupando com as necessidades de cada comunidade. Ao fim de cada um desses componentes curriculares era elaborado um relatório com todas as atividades realizadas nas USF e apresentado para as respectivas equipes de saúde.

A atuação profissional dos cirurgiõesdentistas vêm sofrendo mudanças que enfatizam a necessidade no processo formador de abordagens que não fiquem só na teoria, mas que envolvam a prática e todas as nuances que compõem a atenção básica. Possibilitar esse conhecimento é um desafio durante a formação acadêmica, pois 0 processo ensino- aprendizagem deve sair do modelo centrado no diagnóstico de doenças, tratamento e recuperação para o centrado no diagnóstico integral, promoção de saúde, na prevenção e no cuidado com as pessoas, conforme proposto por Morita e Kriger ${ }^{11}$.

\section{Considerações Finais}

O convívio com a comunidade para a nossa formação acadêmica foi de suma importância, pois para além de aprender um procedimento técnico, a interação com as pessoas da comunidade adstrita às USF nos fez compreender que cada um carrega uma história que se projeta na saúde bucal e geral daquela região. Dessa forma, aprendemos que o atendimento e o planejamento de cada paciente, apesar de individuais, refletem, em sua queixa e na sua perspectiva diante de um resultado, seu contexto histórico social.

\section{Referências}

1. Brasil. Ministério da Saúde; Secretaria de Atenção à Saúde. Departamento de Atenção Básica/Saúde Bucal - Brasília : Ministério da Saúde, 2008. 92 p. - (Série A. Normas e Manuais Técnicos) (Cadernos de Atenção Básica; 17).

2. Brasil. Ministério da Saúde [internet]; Departamento de Atenção básica. Disponível em: http://bvsms.saude.gov.br/bvs/publicacoes/politi ca nacional brasil sorridente.pdf. Acesso em 29 de nov de 2018.

3. Mattos GCM; Ferreira EF; Leite ICG; Greco RM. A inclusão da equipe de saúde bucal na Estratégia Saúde da Família: entraves, avanços e desafios. Ciência \& Saúde Coletiva, 2014; 19(2):373-382.

4. Brasil. Ministério da Saúde [internet]; Departamento de Atenção básica. Disponível em: http://dab.saude.gov.br/portaldab/ape brasil so rridente.php?conteudo=faq. Acesso em 29 de nov de 2018.

5. Brasil. Ministério da Educação [internet]; Conselho Nacional De Educação Câmara De Educação Superior. Disponível em: http://portal.mec.gov.br/cne/arquivos/pdf/CESO 32002.pdf. Acesso em 29 de nov de 2018.

6. Gonçalves GH. A Inserção Dos Acadêmicos De Odontologia Na Estratégia De Saúde Da Família. Disponível em: https://www.nescon.medicina.ufmg.br/bibliotec a/imagem/GIULLIANO-HENRIQUEGON\%C3\%87ALVES.pdf. Acesso em 29 de nov de 2018.

7. Sanchez HF; Drumond MM; Vilaça EL. Adequação de recursos humanos ao PSF: percepção de formandos de dois modelos de formação acadêmica em odontologia. Ciênc. Saúde Coletiva, 2008; 13(2).

8. Almeida FCM; Pessoa MAP; Ramos BA; Barros FC; Ibiapina JR; Souza SMF; Araújo DP. Avaliação da Inserção do Estudante na Unidade Básica de Saúde: Visão do Usuário. Revista Brasileira De Educação Médica. 201236 (1 Supl. 1) : $33-39$.

9. Leme PAT; Pereira AC; Meneghim MC; Mialhe FL. Perspectivas de graduandos em 
odontologia acerca das experiências na atenção básica para sua formação em saúde. Ciência \& Saúde Coletiva, 2015; 20(4):1255-1265.

10. Rodrigues AAAO; Gallotti AP; Pena SFA; Ledo CAS. Saúde Bucal No Programa De Saúde Da Família Na Cidade De Feira De Santana (Ba): O Perfil Do Cirurgião-Dentista. Revista Baiana de Saúde Pública. 2009; 33(4): 582-94.

11. Morita MC, Kriger L. Mudanças nos cursos de Odontologia e a interação com o SUS. Rev Abeno. 2004; 4(1):17-21.

\section{Endereço para Correspondência}

Universidade Estadual de Feira de Santana

Avenida Transnordestina, s/n - Novo Horizonte

Feira de Santana - Bahia

CEP.: 44036-900

aaaorodrigues@gmail.com

Recebido em 22/09/2020

Aprovado em 13/02/2021

Publicado em 06/04/2021 